\title{
Wie bewähren sich GLP-1-Analoga in der ärztlichen Praxis?
}

Schon seit etwa 4 Jahren hat Dr. Jörg Lüdemann, Falkensee, Therapieerfahrungen mit dem humanen-GLP-1-Analogon Liraglutid gesammelt - er war bereits an der LEAD-6Studie, dem direkten Vergleich von Liraglutid und Exenatid, beteiligt. Seit der Zulassung des Wirkstoffs im Sommer 2009 steht das Präparat in Kombination mit Metformin und/oder Sulfonylharnstoff für alle Patienten mit Typ-2-Diabetes zur Verfügung. Im Interview in „Diabetes aktuell“ äußerte sich Dr. Jörg Lüdemann zu den Vorteilen bzw. eventuellen Problemen bei der Therapie mit Liraglutid.

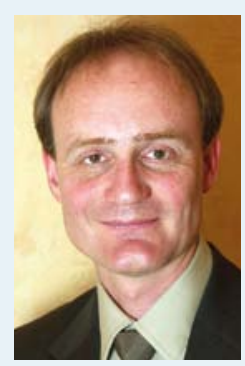

? Wie kommen die Patienten mit der Anwendung des GLP-1-Analogons im Alltag zurecht? Lüdemann: Die Anwendung von Liraglutid im täglichen Leben ist denkbar einfach - es wird einmal pro Tag mahlzeitenunabhängig verabreicht und verursacht keine relevanten Hypoglykämien. Auch die Tatsache, dass Liraglutid injiziert werden muss, stellt keine Hürde für die Patienten dar. So überwiegen für viele Patienten die Vorteile einer sicheren Blutzuckersenkung und Gewichtsreduktion den Aspekt der Spritze deutlich. Allein die Aussicht, mit einem GLP-1Analogon aus dem Teufelskreis von Gewichtszunahme oder sich weiter verschlechternden Blutzuckerwerten ausbrechen zu können, überzeugt die meisten Patienten. Wir sahen daher in der Vergleichsstudie mit dem anderen Inkretinprinzip, den oralen DPP-4-Inhibitoren, unter Liraglutid eine höhere Patientenzufriedenheit [1]. Die Übelkeit als wesentliche Nebenwirkung findet sich nur in geringem Maß und ist schnell rückläufig. In der Vergleichsstudie war nach einigen Wochen kein Unterschied in der Verträglichkeit mehr zu beobachten.

? Welche Rolle spielen Hypoglykämien bei der Anwendung von Liraglutid? Lüdemann: Als Begründung für Patienten, die sich trotz schlechter Blutzuckerwerte und Diabetesfolge-Komplikationen einer Insulintherapie verweigern, wird oft die Spritzenangst herangezogen. Es ist aber eher Angst vor Gewichtszunahme, Hypoglykämien und eingeschränkter Selbständigkeit, die viele Patienten durch die notwendige Insulindosisanpassung und Blutzuckerselbstkontrolle empfinden. Diese Probleme entfallen bei einem GLP-1-Analogon im Wesentlichen.
? Schlägt sich die einfache Anwendung von Liraglutid auch in einer verbesserten Therapietreue der Patienten nieder?

Lüdemann: Die einfache Anwendung, die gute Verträglichkeit sowie die Aussicht, neben einer sicheren Blutzuckersenkung auch das Übergewicht reduzieren zu können, sind starke Motivatoren für diese Therapieform. Anhand der Verschreibungen können wir die hohe Compliance der Patienten im Praxisalltag leicht überprüfen. Dabei zeigt sich, dass Patienten mit Liraglutid die treuesten und zuverlässigsten Anwender sind, die ein großes Interesse daran haben, diese Diabetestherapie so lange wie möglich nutzen zu können.

\section{? Wie relevant ist Ihrer Meinung nach das Thema Gewicht bei der Therapieauswahl und in den Gesprä- chen mit den Patienten?}

Lüdemann: Gerade bei Patienten mit Adipositas ist das Thema Gewicht wichtiger als die reine Blutzuckereinstellung. Viele Patienten haben seit Jahren frustrierende Erfahrungen mit fehlgeschlagenen Diäten und Jo-Jo-Effekten hinter sich. Adipositas ist zudem ein wesentlicher Faktor für Komorbiditäten, wie z.B. Hypertonus, Herz-, Gelenkerkrankungen, Demenz und Krebs. Allerdings empfinden sich dicke Menschen häufig als stigmatisiert und abgewertet. Nun gibt es mit den GLP-1-Analoga für viele eine echte Chance, gute Blutzuckerwerte und eine Gewichtsreduktion zu erreichen. Mit den Anfangserfolgen erwachen bei vielen Patienten Selbstwertgefühl und Motivation neu, durch Veränderung von Ernährung und Bewegung nicht nur weiter das Gewicht zu reduzieren, sondern sich auch fitter zu fühlen. So sehen wir z. B. auch in den Klonoff-Daten bei einer Therapie mit Exenatid über 3 Jahre einen progredienten Gewichtsverlust bei stabiler Blutzuckereinstellung [2].
? Patienten mit Typ-2-Diabetes weisen häufig gleichzeitig kardiovaskuläre Komorbiditäten und/oder eine Hypertonie auf. In welchem Umfang beeinflusst dies Ihre Therapieentscheidung?

Lüdemann: Nach den Ergebnissen von ACCORD, ADVANCE und VADT und weiteren Studien, wie einer Registerarbeit an britischen Hausarztpraxen, kristallisiert sich heraus, dass Hypoglykämien und Gewichtszunahme die kardiovaskuläre Mortalität verschlechtern [3]. So werden inzwischen abgestufte Zielwerte der Diabeteseinstellung diskutiert. Während bei einem neudiagnostizierten Patienten mit Typ-2-Diabetes ohne koronare Herzerkrankung der $\mathrm{HbA}_{1 \mathrm{c}}$ weiter unter 6,5\% eingestellt werden sollte, ist man bei Älteren und Patienten mit makrovaskulären Folgeerkrankungen bezüglich der Zielwerte deutlich vorsichtiger.

Allerdings gibt es z.B. aus der ACCORDStudie Hinweise, dass auch ältere Patienten mit Typ-2-Diabetes, die mit einem Inkretin-Therapeutikum behandelt wurden, eine deutliche Reduktion der kardiovaskulären Mortalität zeigten. Zudem senken GLP-1-Analoga gewichtsunabhängig den Blutdruck und verbessern die Blutfette. So scheinen gerade bei Patienten mit Komorbiditäten oder im Alter die sicheren Inkretin-Therapeutika ohne Hypoglykämien von besonderem Nutzen zu sein.

\section{? Was erwarten Sie für die Zukunft der Inkretin-Therapeutika?}

Lüdemann: Ob GLP-1-Analoga und DPP-4Inhibitoren die Anzahl kardiovaskulärer Ereignisse senken, wird derzeit in mehreren Endpunktstudien untersucht. Für Liraglutid wurde mit der LEADER $^{\text {TM}}{ }_{\text {-Studie }}$ 2010 ein entsprechendes Programm mit über 9000 Patienten über 5 Jahre gestartet. Ich bin überzeugt, dass diese Studien den Beweis einer Senkung kardiovaskulärer Ereignisse durch GLP-1-Analoga erbringen und die Behandlungsparadigmen verändern werden. Zukünftig werden Inkretine ein wesentlicher Bestandteil der Therapie des Typ-2-Diabetes sein.

Literatur

1 Pratley RE et al. Lancet 2010; 375: 14471456

2 Klonoff DC et al. Curr Med Res Opin 2008; 24: 275-286

3 Currie CJ et al. Lancet 2010; 375: 481-489 


\section{Optimierung der intensivierten Insulintherapie}

Um Hypoglykämien zu vermeiden, halten $50 \%$ der Patienten mit intensivierter konventioneller Insulintherapie (ICT) ihre Blutzuckerwerte bewusst über dem Therapieziel. Hierdurch können sie jedoch nicht von den gesundheitlichen Vorteilen konstant normnaher Blutzuckerwerte profitieren. „Es ist wichtig, eine gute Blutzuckereinstellung mit möglichst wenigen Schwankungen zu erreichen“, betont auch Dr. Hans-Martin Reuter, niedergelassener Diabetologe aus Jena. Das neue codierfreie Blutzuckermesssystem OneTouch ${ }^{\circledR}$ Verio ${ }^{\circledR}$ Pro bietet Diabetespatienten Messgenauigkeit da, wo es darauf ankommt - bei niedrigen und hohen Blutzuckerwerten und überall dazwischen. Zusätzlich bietet das Messsystem eine Funktion zur Optimierung der Insulintherapie: Die Hoch/Niedrig Trenderkennung. Dabei informiert das Messsystem den Patienten automatisch über hohe und niedrige Trends der Blutzuckerergebnisse, sodass er eine Insulinanpassung in Erwägung ziehen kann.

\section{Messgenauigkeit da, wo es darauf ankommt}

In das schnell arbeitende und codierfreie Messsystem ist die GDH-FAD-Enzymtechnologie (Glukose-Dehydrogenase-Flavinadenindinucleotid) mit hoher Substratspezifität integriert. Die OneTouch ${ }^{\circledR}$ GlucoFilter $^{\circledR}$ Technologie liefert in nur 5 Sekunden labornahe Messergebnisse. „Patienten mit einer intensivierten Insulintherapie, bei denen die Gefahr von Hypoglykämien besonders hoch ist, können von einer höheren Genauigkeit von Blutzuckermesssystemen profitieren“, so Reuter. „Denn je genauer der Messwert, desto besser können sie abschätzen, wie viel Insulin sie benötigen“.

\section{Zusätzliche Sicherheit durch}

Hoch/Niedrig-Trenderkennung

Neben der labornahen Messgenauigkeit bietet das neue Messsystem noch einen
OneTouch ${ }^{\circledast}$ Verio ${ }^{\circledast}$ Pro von LifeScan - entwickelt für die Bedürfnisse von Insulinpatienten, die regelmäßig eigene Entscheidungen zur Insulinanpassung treffen.

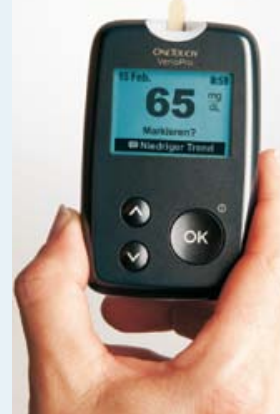

weiteren Vorteil: Die Hoch/Niedrig Trenderkennung, eine Funktion, die dem Patienten hilft, die Insulintherapie zu optimieren. Das Messsystem erkennt automatisch wichtige Blutzuckermuster und informiert den Patienten über hohe und niedrige Blutzuckertrends. So kann er erwägen, ob eine Insulinanpassung notwendig ist. Zusätzlich bietet das System die Funktion, Messwerte zu markieren nüchtern, vor der Mahlzeit, nach der Mahlzeit und Schlafenszeit. Mittelwerte für 7, 14, 30 und 90 Tage werden übersichtlich dargestellt.

Pressemitteilung LifeScan

\section{Blutzuckerselbstkontrolle erleichtert Management}

Bei der Behandlung des Diabetes mellitus spielt die Mitarbeit und Therapieadhärenz des Patienten eine wichtige Rolle. Ohne ein erhebliches Maß an Selbstdisziplin und der Bereitschaft den Lebensstil an den Verlauf der Erkrankung anzupassen, ist der Krankheitsverlauf nur schwer beherrschbar. Insbesondere das wichtige Ziel einer normnahen Stoffwechseleinstellung wird dann nicht erreicht.

„Die praktikabelste Methode zur Überwachung der Blutglukose und zur Vermeidung von Stoffwechselentgleisungen ist die Blutzuckerselbstkontrolle (SelfMonitoring of Blood Glucose = SMBG)“, so Prof. Peter Schwarz, Dresden. Voraussetzungen für den erfolgreichen Einsatz der Blutzuckerselbstkontrolle im Rahmen des Diabetes-Selbstmanagements sind die einfache Handhabung des Blutzuckermessgeräts sowie eine strukturierte Anwendungsschulung zur Vermeidung von Messfehlern. Aber auch die objektive Beweisführung der Blutzuckerwerte ist von großer Bedeutung, bezieht der behandelnde Arzt doch aus den dokumentier- ten Messwerten entscheidende Hinweise für seine Behandlungsstrategie.

\section{Mit Blutzuckerselbstkontrolle und} Datenmanagement mehr Bereitschaft zur Verhaltensänderung

Die Blutzuckermessung mit dem Contour $^{\circledR}$ USB mit integrierter Diabetes-Management-Software liefert solche Daten in übersichtlichen Grafiken und Charts und erleichtert auf diese Weise die Kontrolle der Blutzuckerwerte und das Erkennen von beispielsweise wiederkehrenden metabolischen Krisen. Durch ein entsprechendes Feedback von Arzt oder Diabetesberaterin besteht die Chance auf eine Steigerung der Motivation zur dauerhaften Verhaltensänderung, die Voraussetzung für ein erfolgreiches Selbstmanagement ist.

Die Kombination aus Blutzuckermessgerät und integrierter Diabetes-Management-Software führt zu einer insgesamt verbesserten Diabetesbehandlung auch im Alltag von insulinpflichtigen Typ-1und Typ-2-Diabetikern, wie eine nicht interventionelle, multizentrische Beobachtungsstudie ergab. Die Verwendung des Contour ${ }^{\circledR}$ USB Systems führte zu einer besseren Compliance, wie $70 \%$ der befragten Ärzte feststellten. Fast 60\% der Studienteilnehmer konnten Stoffwechselentgleisungen leichter identifizieren und knapp $72 \%$ bestätigten eine bessere Kontrolle der Blutzuckereinstellung ihrer Patienten. Im Rahmen einer Subgruppenanalyse bei 237 Patienten konnte auch ein positiver Einfluss auf untersuchte Laborparameter, die sowohl bei der Aufnahme- als auch bei der Abschlussuntersuchung erhoben wurden, nachgewiesen werden. So sank der Nüchternblutzucker durchschnittlich um $11,2 \mathrm{mg} / \mathrm{dl}$, der postprandiale Blutzuckerspiegel um 19,2 mg/ dl und der $\mathrm{HbA}_{1 \mathrm{c}}$-Wert um 0,4 Prozentpunkte.

Richard Kessing, Zeiskam

Quelle: „Diabetes managen- Folgen reduzieren“ Pressegespräch im Rahmen der 46. Jahrestagung der Deutschen Diabetes Gesellschaft (DDG) am

1. Juni 2011 in Leipzig. Veranstalter: Bayer Health Care, Bayer Vital, Leverkusen. 